\title{
Estimation of the Rigid-Body Motion from Three-Dimensional Images Using a Generalized Center-of-Mass Points Approach
}

\author{
B. Feng [Member, IEEE], P. P. Bruyant [Member, IEEE], P. H. Pretorius [Associate Member], \\ R. D. Beach [Senior Member, IEEE], H. C. Gifford [Member, IEEE], J. Dey [Member, IEEE], M. \\ Gennert, and M. A. King [Senior Member, IEEE] \\ B. Feng, P. P. Bruyant, P. H. Pretorius, R. D. Beach, H. C. Gifford, J. Dey, and M. A. King are with the \\ Department of Radiology, University of Massachusetts Medical School, Worcester, MA 01655. M. Gennert \\ is with the Department of Computer Science, Worcester Polytechnic Institute, Worcester, MA.
}

\section{Abstract}

We present an analytical method for the estimation of rigid-body motion in sets of three-dimensional SPECT and PET slices. This method utilizes mathematically defined generalized center-of-mass points in images, requiring no segmentation. It can be applied to compensation of the rigid-body motion in both SPECT and PET, once a series of 3D tomographic images are available. We generalized the formula for the center-of-mass to obtain a family of points co-moving with the object's rigid-body motion. From the family of possible points we chose the best three points which resulted in the minimum root-mean-square difference between images as the generalized center-of-mass points for use in estimating motion. The estimated motion was used to sum the sets of tomographic images, or incorporated in the iterative reconstruction to correct for motion during reconstruction of the combined projection data. For comparison, the principle-axes method was also applied to estimate the rigid-body motion from the same tomographic images. To evaluate our method for different noise levels, we performed simulations with the MCAT phantom. We observed that though noise degraded the motion-detection accuracy, our method helped in reducing the motion artifact both visually and quantitatively. We also acquired four sets of the emission and transmission data of the Data Spectrum Anthropomorphic Phantom positioned at four different locations and/or orientations. From these we generated a composite acquisition simulating periodic phantom movements during acquisition. The simulated motion was calculated from the generalized center-of-mass points calculated from the tomographic images reconstructed from individual acquisitions. We determined that motioncompensation greatly reduced the motion artifact. Finally, in a simulation with the gated MCAT phantom, an exaggerated rigid-body motion was applied to the end-systolic frame. The motion was estimated from the end-diastolic and end-systolic images, and used to sum them into a summed image without obvious artifact. Compared to the principle-axes method, in two of the three comparisons with anthropomorphic phantom data our method estimated the motion in closer agreement to than of the Polaris system than the principal-axes method, while the principle-axes method gave a more accurate estimation of motion in most cases for the MCAT simulations. As an image-driven approach, our method assumes angularly complete data sets for each state of motion. We expect this method to be applied in correction of respiratory motion in respiratory gated SPECT, and respiratory or other rigid-body motion in PET.

(telephone: 508-856-8795, e-mail: Bing.Feng@umassmed.edu). (email: Michaelg@sequoia.wpi.edu). 


\section{Introduction}

In SPECT and PET, patient motion during imaging causes reconstruction artifacts and degrades diagnostic accuracy if not correctly compensated [1-4]. There are numerous papers published on this subject, and more can be expected. Based on the motion-detection technique employed, these papers can be divided into the categories of data-driven methods [5-12] and external tracking system methods [13-21]. Each category of methods have advantages and disadvantages. The external tracking system methods can employ an IR tracking device such as the Polaris, or a video-tracking system (VTS) to detect the motion of isolated markers. This offers high accuracy when estimating the motion of markers, but in some cases (for example respiratory motion) does not directly reflect the motion of the organ imaged. The data-driven methods estimate the motion from the data and may use iterative approaches based on various objective functions. Converging or robust algorithms are not easy to obtain.

We propose a simple analytical method of detecting rigid-body motion from a series of sets of three-dimensional (3D) tomographic images. Other analytical algorithms for rigid-body registration have been published and successfully applied in the medical imaging field. Examples of such analytical methods include the use of tensor-based moment functions [22], and the principle-axes method $[23,24]$. Our method is a generalization of the conventional center-of-mass approach which gives only information for rigid-body translation [25]. For estimating rotation in addition to translation we defined mathematically a family of "generalized center-of-mass points". The motion of these points matches the motion in the images. These generalized points can be used to correct rigid-body motion in image reconstruction, as in the case of using the rigid-body motion detected by an external tracking system [15-17]. One distinction between our method and the methods utilizing the tensor-based moment functions or the principle-axes method is that the generalized center-of-mass points are linear in terms of the coordinates, while the other methods mentioned use tensors which are non-linear in terms of the coordinates. An additional feature of our method is the optimization process, implemented to choose the power functions and pre-smoothing Gaussian functions which lead to the optimal alignment between two sets of images. For comparison, the principle-axes method [23] was also implemented and applied to our test data.

One limitation of our method is that a series of three-dimensional tomographic images, one for each state of motion of the object, must be available. Usually a SPECT acquisition cannot provide such a series of tomographic images. However, in a respiratory gated acquisition, one set of images can be reconstructed for each stage of respiration. In PET, it is possible to divide acquisition data into independent time bins and thereby reconstruct a series of images [26]. Our method might be applied to the above cases. In reality respiratory motion has been shown to be complex and non-linear [27], we expect our method could be employed to compensate for the rigid-body part of the respiratory motion affecting the heart.

\section{Methods}

\section{A. Detection of the Rigid-Body Motion from the Generalized Center-of-Mass Points and by the Principle-Axes Method}

We assume a series of tomogaphic reconstructions of a moving object are available, from which the rigid-body motion of the object can be determined. Each of the reconstructions describes the $3 \mathrm{D}$ intensity distribution of the object at a certain time. The $\mathrm{i}^{\text {th }} \operatorname{voxel}\left(i=1,2, \ldots, N_{p}\right.$, where $N_{p}$ is the number of voxels) has intensity $m_{i}$. From time $\mathrm{t}$ to $\mathrm{t}+\mathrm{dt}$, we assume that the center of the $\mathrm{i}^{\text {th }}$ voxel $m_{i}$ moves from coordinates $\left(x_{i}, y_{i}, z_{i}\right)$ to $\left(\tilde{x}_{i}, \tilde{y}_{i}, \tilde{z}_{i}\right)$, due to a rigid-body 
motion. The coordinate transformation for this is $\left[\begin{array}{c}\tilde{x}_{i} \\ y_{i} \\ z_{i}\end{array}\right]=[M]\left[\begin{array}{c}x_{i}-x^{o} \\ y_{i}-y^{o} \\ z_{i}-z^{o}\end{array}\right]+\left[\begin{array}{c}x^{o}+\Delta x \\ y^{o}+\Delta y \\ z^{o}+\Delta z\end{array}\right]$, where $[M]$ is the rotation matrix, $\left(x^{o}, y^{o}, z^{o}\right)$ is the center of rotation, and $\left[\begin{array}{c}\Delta x \\ \Delta y \\ \Delta z\end{array}\right]$ is the translation vector. We define generalized center-of-mass points at time $t$ as

$$
\left[\begin{array}{c}
x_{c}^{f} \\
y_{c}^{f} \\
z_{c}^{f}
\end{array}\right]=\frac{\sum_{i=1}^{N_{p}}\left\{f\left(m_{i}\right)\left[\begin{array}{c}
x_{i} \\
y_{i} \\
z_{i}
\end{array}\right]\right\}}{\sum_{i=1}^{N_{p}} f\left(m_{i}\right)}
$$

where function $f\left(m_{i}\right)$ gives a cost to $m_{i}$, and a set of functions gives a set of points. The conventional center-of-mass point uses $f\left(m_{i}\right)=m_{i}$. Higher order moments of $m_{i}$ can be used to provide sets of functions $f\left(m_{i}\right)$. Note that the definition is linear with coordinates $\left(x_{i}, y_{i}\right.$, $z_{i}$ ). At time $\mathrm{t}+\mathrm{dt}$, the generalized center-of-mass points are

$$
\left[\begin{array}{c}
\tilde{x}_{c} \\
\tilde{c}_{c} \\
y_{c} \\
\tilde{z} \\
z_{c}
\end{array}\right]=\frac{\sum_{i=1}^{N_{p}}\left\{f\left(m_{i}\right)\left[\begin{array}{c}
\tilde{x}_{i} \\
\tilde{y}_{i} \\
\tilde{z} \\
z_{i}
\end{array}\right]\right\}}{\sum_{i=1}^{N_{p}} f\left(m_{i}\right)} .
$$

Keeping in mind that the rotation matrix and translation vector consist of unknown constants,

it is straightforward to prove $\left[\begin{array}{c}v_{c}^{f} \\ x_{c} \\ y_{c} \\ z_{c}^{f} \\ z_{c}\end{array}\right]=[M]\left[\begin{array}{c}x_{c}^{f}-x^{o} \\ y_{c}^{f}-y^{o} \\ z_{c}^{f}-z^{o}\end{array}\right]+\left[\begin{array}{c}x^{o}+\Delta x \\ y^{o}+\Delta y \\ z^{o}+\Delta z\end{array}\right]$, which means that the generalized center-of-mass points have the same rigid-body motion as the object. Function $f$ $\left(m_{i}\right)$ should be chosen under the constraint of $f(0)=0$, otherwise it leads to change of the image support and truncation of the transformed image $f\left(m_{i}\right)$. One simple choice of $f\left(m_{i}\right)$ that satisfies $f(0)=0$ is a power function as employed herein.

We need at least three generalized center-of-mass points in each image to track 6-degree-offreedom rigid-body motion. This is an analog to the case of fixing an object in 3D where you need at least three pins. That is, one point can determine translation, and a second can fix two rotational degrees of freedom about the first. However a third point is needed to establish the third rotational degree-of-freedom. By choosing the function $f(m)$ in Equation (1) as power functions $f_{j}(m)=m^{j}(\mathrm{j}=1,2,3, \ldots, \mathrm{N}), \mathrm{N}$ generalized center-of-mass points are obtained. Generally different points will be obtained for different power functions, since the higher the power function index, the closer the generalized center-of-mass point is to the hottest point in the image. Since more than three points are available, we optimize the method by using the "best" three points. The criterion employed is the root-mean-square difference (RMSD) between the image at time $t+d t$ and the image at time $t$ with its location moved by the rigidbody motion that is determined from three of $\mathrm{N}$ points. Once any three points (characterized by the indexes of power functions) are chosen, we employ a least-squares algorithm [28] that uses singular value decomposition (SVD) to estimate the rigid-body motion. The RMSD is calculated by 


$$
R M S D=\sqrt{\frac{\sum_{i=i}^{N_{p}}\left(m_{i}^{t+d t}-\widehat{m}_{i}^{t}\right)^{2}}{N_{p}}},
$$

where $N_{p}$ is the number of voxels in each image, $m_{i}^{t+d t}$ is the image intensity at $\mathrm{i}^{\text {th }}$ voxel measured at time $\mathrm{t}+\mathrm{dt}$, and $\widehat{m}_{i}^{t}$ is the image intensity at the same voxel on the image that is measured at time $\mathrm{t}$ and shifted with the estimated rigid-body motion. Note that both $m_{i}^{t+d t}$ and $\widehat{m}_{i}^{t}$ represent the original unsmoothed images. RMSD is dependent on the estimated rigid-body motion, therefore it is dependent on the power functions chosen to generate the generalized center-of-mass points. The time for optimization was approximately proportional to $\left(\begin{array}{c}3 \\ N\end{array}\right)=N(N-1)(N-2) / 6$ the optimization significantly. . $\mathrm{N}$ was chosen as 5 in our study since a higher $\mathrm{N}$ would slow down

Generally images are degraded by noise which is caused by the random nature in the imaging process. Therefore we investigated whether it was preferable to apply pre-smoothing to the images before calculation of the generalized center-of-mass points. The pre-smoothing function we chose is a three-dimensional Gaussian function, whose sigma is chosen from a few discrete values (from 0 up to 5.5 pixels with a step size of 0.5 pixel). The optimal presmoothing Gaussian function is chosen as the one that gives the minimum RMSD.

By minimizing RMSD, the rigid-body motion is estimated from images at times $t$ and $t+d t$. In the case of more than two time points, a baseline configuration is chosen (for example, the first time point), and the motion from the baseline can be estimated for other time points. The estimated motion can be used post-reconstruction to generate a single summed set of slices from the series of slices for different states of motion. Once the motion is estimated from the set of 3D tomographic slices, it can also be used to model the motion in reconstruction of all of the projection data as a whole dataset [20]. In this case, the reconstruction goes through all the motion states at each projection angle modeling the motion relative to some reference position. These two motion compensation strategies (intelligent summing and modeling motion in reconstruction) will both be evaluated through the MCAT simulations and experimental measurements.

The principle-axes [23] method was also implemented to provide a comparison of the generalized center-of-mass approach to an existing method. Based on the classical theory of the rigid-body motion, the principle-axes were defined as the eigenvectors of the inertia tensor. Defined in Equation (9) in Faber et al [23], the inertia tensor is symmetric and its eigenvectors are orthogonal to each other if the three eigenvalues are non-degenerate. These orthogonal principle-axes rotate with the object in the case of the rigid-body rotation. The rotation matrix $[M]$ is equal to the sum of the tensor products of the unit eigenvectors (denoted by $v_{1}, v_{2}, v_{3}$ ) at time $\mathrm{t}$ (before motion) and their counterparts (denoted by $\tilde{v}_{1}, \widetilde{v}_{2}, \tilde{v}_{3}$ ) at time $\mathrm{t}+\mathrm{dt}$. To write explicitly,

$$
[M]=\tilde{v_{1}} \otimes v_{1}+\tilde{v}_{2} \otimes v_{2}+\tilde{v}_{3} \otimes v_{3},
$$

where $\otimes$ denotes the tensor product of two vectors. For each eigenvector, there is freedom of going positive or negative direction. Eigenvectors $v_{1}, v_{2}, v_{3}$ and $\widetilde{v}_{1}, \widetilde{v}_{2}, \widetilde{v}_{3}$ are chosen to form right-handed sets. In addition, the angles between $v_{1}$ and $\tilde{v}_{1}$, between $v_{2}$ and $\tilde{v}_{2}$ are forced to be no greater than 90 degrees to avoid ambiguity. Since for any vectors $a, b$, and $c$, we have

$$
(a \otimes b) \bullet c=(b \bullet c) a,
$$

where $\bullet$ denotes the dot product. It is simple to show that 


$$
[M] \bullet v_{1}=\tilde{v}_{1},[M] \bullet v_{2}=\tilde{v_{2}},[M] \bullet v_{3}=\tilde{v}_{3},
$$

which means that $[M]$ rotates $v_{1}, v_{2}, v_{3}$ to $\tilde{V}_{1}, \widetilde{V}_{2}, \widetilde{V}_{3}$, respectively.

The translation was calculated from the center-of-masses at time $t$ and time $t+d t$.

\section{B. MCAT Phantom Simulations with Different Noise Levels}

To experimentally investigate our method under controlled conditions we employed an MCAT phantom which contained only the heart and liver as sources. We created two configurations: (1) before the motion (at time t) and (2) after the motion (at time $t+d t)$. The motion consisted of a 15-degree rotation around the vertical (Y) axis and a translation ( $\Delta x=2 \mathrm{~cm}, \Delta y=3 \mathrm{~cm}$ and $\Delta z=2.5 \mathrm{~cm}$ ). The projection data were generated for the two configurations by using a ray-driven projector which modeled attenuation and distance-dependent blurring. Each set of projection data contained 60 frames, simulating a 180-degree acquisition on a SPECT system of a Tc-99m perfusion imaging-agent. Four noise-levels were simulated: noise-free, 1 million (M), $0.5 \mathrm{M}$ and $0.1 \mathrm{M}$ counts in the heart, with Poisson noise added. This resulted in 4 motionfree datasets for each configuration. After reconstructing these datasets with compensations for attenuation and distance-dependent blurring, we calculated the generalized center-of-mass points on the reconstructed images, with optimization of the pre-smoothing Gaussian function and the three power functions in terms of the minimum RMSD. The estimated motion was used to move the image at time $\mathrm{t}+\mathrm{dt}$ back and sum it into the image at time $t$. Three-dimensional Gaussian interpolation [20] was employed to move images. To test usefulness of derived motion in an iterative reconstruction with motion compensation, 4 other datasets (for 4 noise levels, respectively) were created by combining data of the two configurations, simulating an acquisition of a periodic motion which consisted of only two motion states (at each angle the phantom moved between configurations 1 and 2 back and forth). Using the OSEM algorithm which models the known rigid-body motion with the 3D Gaussian interpolation [20], we reconstruct the motion-present datasets with the motion derived from the generalized centerof-mass points. To evaluate the quantitative accuracy of the reconstruction, we calculated the root-mean-square error (RMSE) which has an expression similar to Equation (3). In other words, the RMSE was defined as the RMSD between a certain reconstruction and the truth. For each noise-level, the truth was assumed to be the image reconstructed with compensation for the true motion.

The principle-axes method was also applied to the images at $t$ and $t+d t$ which had been reconstructed from the motion-free datasets to allow comparison to this established method. The rigid-body motion obtained from the principle-axes method was substituted into Equation (3) to calculate the RMSD.

\section{Experimental Study with an Anthropomorphic Phantom}

We also acquired four datasets of the Data Spectrum anthropomorphic phantom with cardiac insert on an IRIX system. One $\mathrm{mCi}, 10.2 \mathrm{mCi}$, and $6.3 \mathrm{mCi}$ of Tc-99m was added into heart, liver, and body of the phantom, respectively. This resulted in approximate concentrations of 1.0, 0.1, and 1.0 in these three structures. A Polaris (Northern Digital Inc.) tool consisting of four spherical markers was attached tightly to the top of the anthropomorphic phantom. Between each acquisition the phantom was translated and or rotated. The orientation was recorded by Polaris. In each acquisition, two heads of the IRIX acquired a total of 204 degree data with 102 degree gantry rotation at 34 angular steps. A scatter window (5\% width centered at $120 \mathrm{keV}$ ) was also acquired. After reconstructing each individual acquisition, we calculated the generalized center-of-mass points and the rigid-body motion, by optimizing the presmoothing Gaussian function and determining which three power functions to be employed based on minimizing the RMSD. Summed images were created with and without taking into 
account the motions. A mixed dataset was generated to simulate an acquisition in which the phantom moved periodically at each angle in an exaggerated "respiratory motion" manner. We reconstructed the mixed dataset with and without modeling the motion in the iterative reconstruction.

The principle-axes method was again utilized to estimate the rigid-body motions in the images reconstructed from the four acquisitions to provide a comparison to our method.

\section{Estimation of the Rigid-Body Motion in Presence of Non-Rigid-Body Motion}

The end-diastolic (ED) and end-systolic (ES) frames of a gated MCAT phantom [29] were chosen for this study. Only the heart (the left and right ventricles) of the phantom was used in our test. The cardiac motion between the ED and ES frames consisted of shortening of the left ventricle (LV) and thickening of the LV wall. The shape of the heart changed from the enddiastole to the end-systole, thus the cardiac motion in the gated MCAT phantom was a nonrigid-body motion. We applied an exaggerated translation ( $\Delta x=2 \mathrm{~cm}, \Delta y=3 \mathrm{~cm}$ and $\Delta z=$ $2.5 \mathrm{~cm}$ ) and rotation (15 degrees around Y-axis) to the ES image. The motion between the ED and moved ES was estimated with the generalized center-of-mass points approach. The estimated motion was compensated in summing the ES image into ED image. It should be noted that this will not result in a full correction as only a rigid-body correction is being made of a motion which is in part non-rigid. However, this serves as a test of correction of the rigidbody component of motion when there is a non-rigid component also present. For comparison, a directly summed image was also generated without compensating for the rigid-body motion. Also to serve as a comparison, the principle-axes method was applied to estimate the rigidbody motion between the ED image and the moved ES image.

\section{Results}

\section{A. MCAT Phantom Simulations with Different Noise Levels}

In the MCAT simulations, the rigid-body motion was estimated from the images before and after the motion. In calculation of the generalized center-of-mass points and estimation of the rigid-body motion, the optimal pre-smoothing Gaussian function and the optimal three power functions were chosen based on the minimum RMSD defined in Equation (3). The results for the optimal parameters and estimated motion are listed in Table 1. As the counts decreased, the accuracy in motion estimation also decreased. This was also reflected as an increase in the minimum relative RMSD for the image with lower counts. The relative RMSD is the RMSD scaled by the counts.

Applied to the same images, the principle-axes method gave more accurate estimates of the rigid-body motion for all the four noise-levels (Table 2). The motion-corrected summed images using the principle-axes method show no visual difference from those images using our method. However, the RMSD from the principle-axes method was significantly larger for each noiselevel than the RMSD from the generalized center-of-mass points method. In simulations of the projection data, attenuation and distance-dependent blurring were modeled in the numerical projector. These degradation factors might have affected the accurate reconstruction of the images and thus the determination of the motion. The smaller RMSD from our method could also be the result of the optimization process. The generalized center-of-mass points method being selected on the basis of minimizing the RMSD as opposed to minimizing the error in the estimate of the motion. The smaller the RMSD, the better the overall alignment of the two sets of images, and the less severe motion artifact in the summed image. Thus this was selected as our criterion for optimization of the parameters of the generalized center-of-mass points method. 
Due to the optimization process involved, our method was slower than the principle-axes method. On a 2.8-GHZ Xeon computer running Linux, the time to align two sets of $128 \times 128 \times 128$ images was 14 seconds for the principle-axes method, and 50 seconds for the generalized center-of-mass points approach.

The motion estimated from the generalized center-of-mass points was used to move the image at time $\mathrm{t}+\mathrm{dt}$ back and sum it with the image at time $\mathrm{t}$ (Fig. 1). As shown in Figure 1, the motion compensated summing generated artifact-free images. To better differentiate different noiselevels, no post-smoothing was applied to the summed images.

The motions estimated from the generalized center-of-mass points were also compensated in the iterative reconstruction of the motion-present datasets. Modeling the motion in reconstruction greatly reduced the motion artifact, compared with reconstruction with no motion compensation (Fig. 2). Using the true-motion compensated images (top row in Fig. 2) as the truth, the relative (scaled by counts) RMSE is listed in Table 3.

\section{B. Experimental Study with an Anthropomorphic Phantom}

The four sets of data were reconstructed using 6 iterations of the OSEM algorithm. The first measurement was chosen as the baseline. Motion from the baseline was estimated by the generalized center-of-mass points method and by the principle-axes method in the reconstructed image for other three cases (Table 4). In Table 4 it can be seen that the generalized center-of-mass points method more closely approximated the motion as estimated by Polaris than the principle-axes method in datasets 2 and 4 . Note that there were sub-pixel translations unreported in Table 4 for datasets 3 and 4 The motions estimated from the generalized centerof-mass points method were used to generate a summed image (Fig. 3) or compensate the motion in the reconstruction of the motion-present data (Fig. 4). In each approach, compensation for the rigid-body motion greatly reduced the motion artifact, as shown on the polar maps in Figure 5.

\section{Estimation of the Rigid-Body Motion in Presence of Non-Rigid-Body Motion}

We applied our method and the principle-axes method to the ED and ES (with an exaggerated rigid-body motion) images. The motion estimated from the generalized center-of-mass points was $2.47,2.93$, and $1.99 \mathrm{~cm}$ translations along $\mathrm{X}, \mathrm{Y}$, and $\mathrm{Z}$ direction, and 11.6 degree rotation by $\mathrm{Y}$-axis. The motion estimated by the principle-axis method was $2.47,3.44$, and $3.14 \mathrm{~cm}$ translations along $\mathrm{X}, \mathrm{Y}$, and $\mathrm{Z}$ direction, and 15.8 degree rotation by $\mathrm{Y}$-axis. The difference between the estimated motion and the true motion $(\Delta x=2 \mathrm{~cm}, \Delta y=3 \mathrm{~cm}, \Delta z=2.5 \mathrm{~cm}$, and a 15 degree rotation by Y-axis) might have been caused partially by the original displacement between the ED and ES images. If the original displacement between the ED and ES images was ignored, our method estimated the translations more accurately than the principle-axes method, while the principle-axes method gave more accurate rotation than our method. The summed images with and without taking into account the rigid-body motion estimated from our method were plotted in Figure 6. Summing with motion compensation greatly reduced the motion artifact.

\section{Discussion}

Our method of using the generalized center-of-mass points reduces the registration of sets of images to the registration of sets of points. However, this method could be sensitive to the noise and other imperfectness present in images, such as artifacts caused by lack of modeling the physics of imaging in reconstruction. By minimizing the RMSD between sets of images, our method optimizes the pre-smoothing Gaussian function and chooses the optimal three power functions that give three points used in estimation of the rigid-body motion. We applied 
this method to the images reconstructed with no attenuation compensation, and found it worked quite well for all noise-levels (from $1 \mathrm{M}$ to $0.1 \mathrm{M}$ ) studied in this manuscript. An alternative approach is to use more than three points. We found that using more than three points resulted in very similar results for motion estimation compared to the approach of using the optimal three points which we concentrated on in this study.

We compared our method with the principle-axes method. We found that the principle-axes method in most cases gave more accurate estimate of the rotation than our method. In two of three cases, our method estimated the translations which agreed better with those measured by the Polaris system than the principle-axes method. One possible explanation could be that only the center-of-mass was used to calculate the translation in the principle-axes method, while our method adjusted the estimate using an optimization process.

As a short-coming of the generalized center-of-mass points method, it does not work for the binary images, since only one point can be obtained by Equation (1). The principle-axes method was shown to be able to successfully align binary images [23].

We tested two motion-compensation strategies once the rigid-body was known. One is summing the $3 \mathrm{D}$ reconstructions after correcting the motion in each set post-reconstruction. The second is reconstructing the motion-present data by modeling the motion in the iterative reconstruction process. Both approaches helped reduce the motion artifacts. The summing approach is faster, while the reconstruction approach is slower but might handle the statistics more appropriately. Since iterative reconstruction is a non-linear process, the two approaches generally result in different images. Further investigations are needed to evaluate these two approaches.

In our simulations with the gated MCAT phantom, the rigid-body motion was extracted approximately in the presence of the non-rigid-body wall motion. In realistic clinic studies, different organs might move in different fashions. Segmentation of the region-of-interest will be required before applying our method.

Like the degeneracy encountered in the registration of points, our method could face the problem of degeneracy if symmetry exists in some directions. In the case of existence of the symmetry in one direction, motion along that direction is undermined, but will not cause motion artifacts in that direction.

It is of most interest whether our method could apply to the clinical patient study. In PET, by diving the acquisition time into short intervals, a series of 3D images could be available. Our method could be applied to sum these images up with motion estimated and compensated. As future work, we will investigate application of this method in PET imaging.

\section{Conclusion}

With simulations and experimental measurement we tested the method of using the generalized center-of-mass points to detect and correct the rigid-body motion in imaging, and we observed our method helped in reducing motion artifacts.

\section{Acknowledgements}

The authors would like to thanks the reviewers for their many useful comments which greatly improved this manuscript.

This work was supported by the National Institute for Biomedical Imaging and Bioengineering grant R01 EB001457 and the National Heart, Lung, and Blood grant R01 HL 50349. The contents are solely the responsibility of the authors and do not necessarily represent the official views of the National Institutes of Health. 


\section{References}

1. Botvinick EH, Zhu YY, O'Connell WJ, Dae MW. A quantitative assessment of patient motion and its effect on myocardial perfusion SPECT images. J Nucl Med 1993;34:303-310. [PubMed: 8429354]

2. Prigent FM, Hyun M, Berman DS, Rozanski A. Effect of motion on thallium-201 SPECT studies: a simulation and clinical study. J Nucl Med 1993;34:1845-1850. [PubMed: 8229222]

3. Cooper JA, Neumann PH, McCandless BK. Effect of patient motion on tomographic myocardial perfusion imaging. J Nucl Med 1992;33:1566-1571. [PubMed: 1634955]

4. Bai, C.; Conwell, R. A systematic simulation study of the effects of patient motion on cardiac perfusion imaging using single photon emission computed tomography; Society of Nuclear Medicine $52^{\text {nd }}$ Annual Meeting; Toronto, Canada. June 18-22, 2005; abstract

5. Eisner R, Churchwell A, Noever T, Nowak D, Cloninger K, Dunn D, et al. Quantitative analysis of the tomographic thallium-201 myocardial bullseye display: critical role of correcting for patient motion. J Nucl Med 1988;29:91-97. [PubMed: 3257259]

6. O'Connor MK, Kanal KM, Gebhard MW, Rossman PJ. Comparison of four motion correction techniques in SPECT imaging of the heart: a cardiac phantom study. J Nucl Med 1998;39:2027-2034. [PubMed: 9867136]

7. Leslie WD, Dupont JO, McDonald D, Peterdy AE. Comparison of motion correction algorithms for cardiac SPECT. J Nucl Med 1997;38:785-790. [PubMed: 9170447]

8. Huang SC, Yu DC. Capability evaluation of a sinogram error detection and correction method in computed tomography. IEEE Trans Nucl Sci 1992;39:1106-1110.

9. Arata, LK.; Pretorius, PH.; King, MA. Correction of organ motion in SPECT using reprojection data; Proceedings of 1995 Nuclear Science Symposium; 1996. p. 1456-1460.

10. Lee KJ, Barber DC. Use of forward projection to correct patient motion during SPECT imaging. Phys Med Biol 1998;43:171-187. [PubMed: 9483630]

11. Matsumoto N, Berman DS, Kavanagh PB, Gerlach J, Hayes SW, Lewin HC, et al. Quantitative assessment of motion artifacts and validation of a new motion-correction program for myocardial perfusion SPECT. J Nucl Med 2001;42:687-694. [PubMed: 11337561]

12. Kyme AZ, Hutton BF, Hatton RL, Skerrett DW, Barden LR. Pratical aspects of a data-driven motion correction approach for brain SPECT. IEEE Trans Med Imag 2003;22:722-729.

13. Fulton RR, Eberl S, Meikle SR, Hutton BF, Braun M. A practical 3D tomographic method for correcting patient head motion in clinical SPECT. IEEE Trans Nucl Sci 1999;46:667-672.

14. Lopresti BJ, Russo A, Jones WF, Fisher T, Crouch DG, Altenburger DE, et al. Implementation and performance of an optical motion tracking system for high resolution brain PET imaging. IEEE Trans Nucl Sci 1999;46:2059-2067.

15. Fulton RR, Meikle SR, Eberl S, Pfeiffer J, Constable RT, Fulham MJ. Correction for head movements in positron emission tomography using an optical motion-tracking system. IEEE Trans Nucl Sci 2002;49:116-123.

16. Beach RD, Pretorius PH, Boening G, Bruyant PP, Feng B, Fulton RR, Gennert MA, Nadella S, King MA. Feasibility of stereo-infrared tracking to monitor patient motion during cardiac SPECT imaging. IEEE Trans Nucl Sci 2004;51:2693-2698.

17. Bruyant PP, Gennert MA, Speckert GC, Beach RD, Morgenstern JD, Kumar N, Nadella S, King MA. A robust visual tracking system for patient motion detection in SPECT: Hardware solutions. IEEE Trans Nucl Sci. submitted

18. Boening, G.; Bruyant, PP.; Beach, RD.; Byrne, CL.; King, MA. Motion correction for cardiac SPECT using a RBI-ML partial-reconstruction approach; Proceedings of 2004 IEEE Medical Imaging Conference; M4-6, in press

19. Fulton RR, Hutton BF, Braun M, Ardekani B, Larkin RS. Use of 3D reconstruction to correct for patient motion in SPECT. Phys Med Biol 1994;39:563-574. [PubMed: 15551598]

20. Feng B, Gifford HC, Beach RD, Boening G, Gennert MA, King MA. Use of three-dimensional Gaussian interpolation in the projector/backprojector pair of iterative reconstruction for compensation of known rigid-body motion in SPECT. IEEE Trans Med Imag. in press 
21. Beach, RD.; Feng, B.; King, MA. Determining patient 6-degrees-of-freedom motion from stereo infrared cameras during supine medical imaging; SPIE Medical Imaging Conference; Town and Country Hotel, San Diego, CA, United States. February 11-16, 2006; abstract

22. Cyganski D, Orr JA. Applications of tensor theory to object recognition and orientation determination. IEEE Trans Pattern Anal Mach Intell 1985;PAMI-7:662-673.

23. Faber TL, Stokely EM. Orientation of 3-D structures in medical images. IEEE Trans Pattern Anal Mach Intell 1988;10:626-633.

24. Alpert NM, Bradshaw JF, Kennedy D, Correia JA. The principle axes Transformation - A method for image registration. J Nucl Med 1990;31:1717-1722. [PubMed: 2213197]

25. Bruyant PP, King MA, Pretorius PH. Correction of the respiratory motion of the heart by tracking of the center of mass of thresholded projections: a simulation study using the dynamic MCAT phantom. IEEE Trans Nucl Sci 2002;49:2159-2166.

26. Picard Y, Thompson CJ. Motion correction of PET images using multiple acquisition frames. IEEE Trans Med Imag 1997;16:137-144.

27. Shechter G, Resar JR, McVeigh ER. Displacement and velocity of the coronary arteries: Cardiac and respiratory motion. IEEE Trans Med Imag 2006;25:369-375.

28. Arun KS, Huang TS, Blostein SD. Least-squares fitting of two 3-D point sets. IEEE Trans Pattern Anal Mach Intell 1987;PAMI-9

29. Pretorius PH, King MA, Tsui BMW, LaCroix KJ, Xia W. A mathematical model of motion of the heart for use in generating source and attenuation maps for simulating emission imaging. Phys Med Biol 1999;26:2323-2332. 


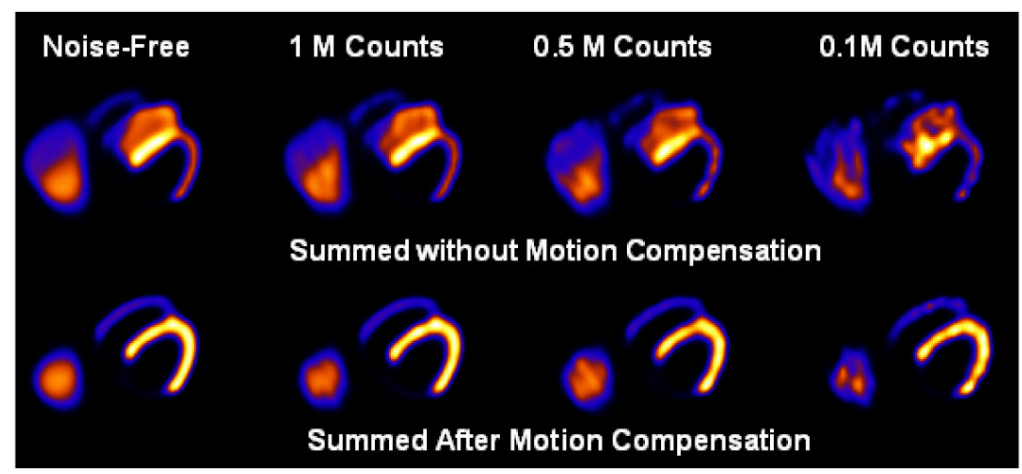

Fig. 1.

Summed images were generated for the MCAT phantom simulations. (Upper row) No motion compensation in summing images at $\mathrm{t}$ and $\mathrm{t}+\mathrm{dt}$. (Lower row) Motion compensated summing. From left to right, results for 4 noise-levels are plotted. No post-smoothing was applied to the summed images. 


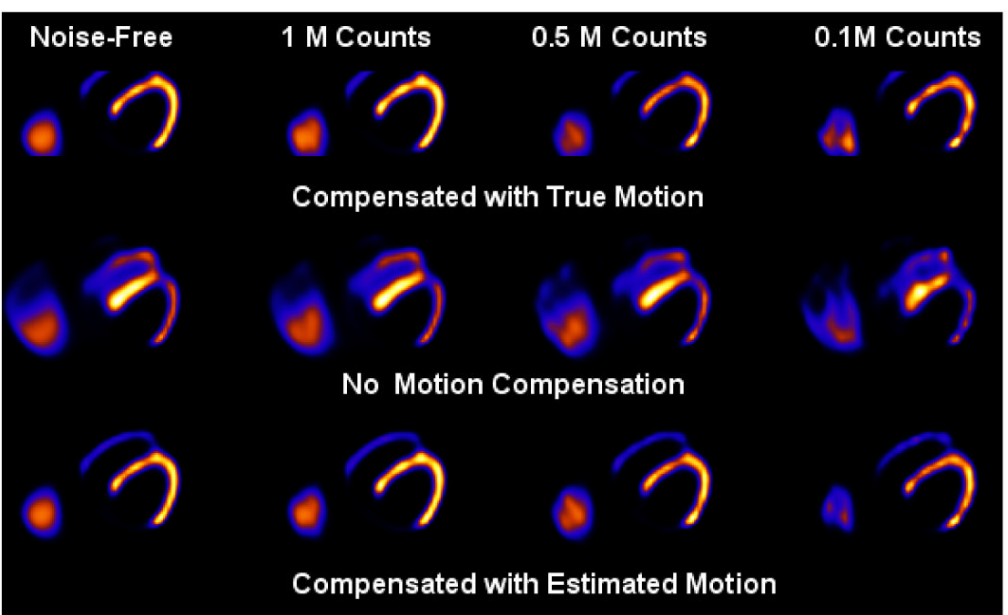

Fig. 2.

Reconstructions of the motion-present simulations for several noise-levels (Bottom row, from left to right), using the motion estimated from the optimal three generalized center-of-mass points (listed in Table 1). As a comparison, the same data were reconstructed with the ideal motion compensation (Upper row) and without motion compensation (Middle row). No postsmoothing was applied to these images. 


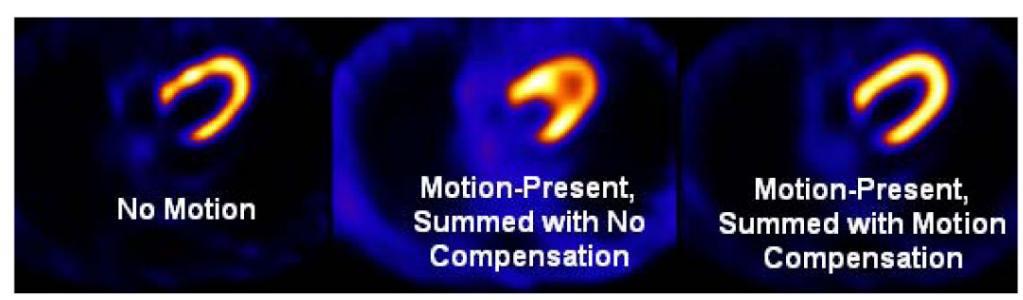

Fig.3.

(Left) Reconstruction of the motion-free baseline acquisition. (Middle) The same transaxial slice for the image summed for the 4 reconstructions of the data acquired in 4 sequential acquisitions, without motion compensation in summing. (Right) The same slice with compensation for motion. No post-smoothing was applied to the images. Note that the summed images had 4 times the counts of the no motion image. 


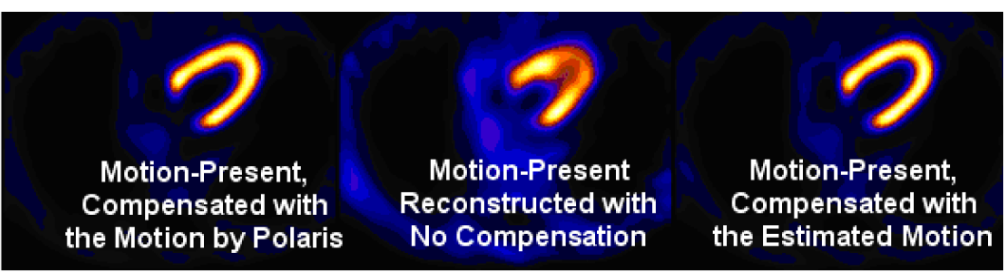

Fig.4.

(Left) Reconstruction of the motion-present data with compensation for the motion which was detected by Polaris. (Middle) No motion Compensation. (Right) Motion compensation with the motion estimated from the generalized center-of-mass points. No post-smoothing was applied to the images. 


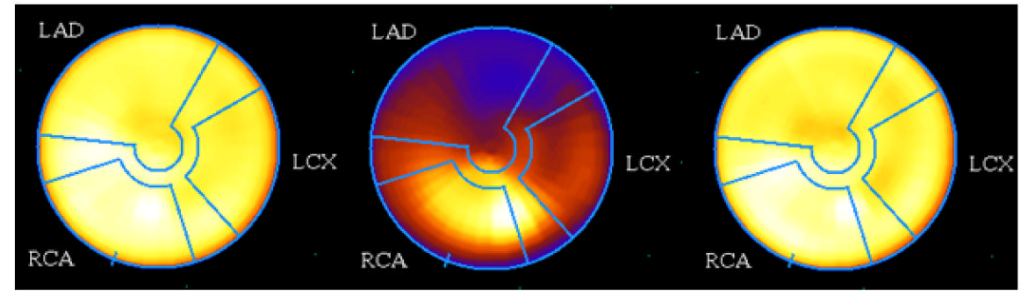

Fig. 5.

Polar maps generated for the images of anthropomorphic phantom shown in Fig. 4. (Left) Motion compensation with Polaris. (Middle) No motion Compensation. (Right) Motion compensation with the generalized center-of-mass points. 


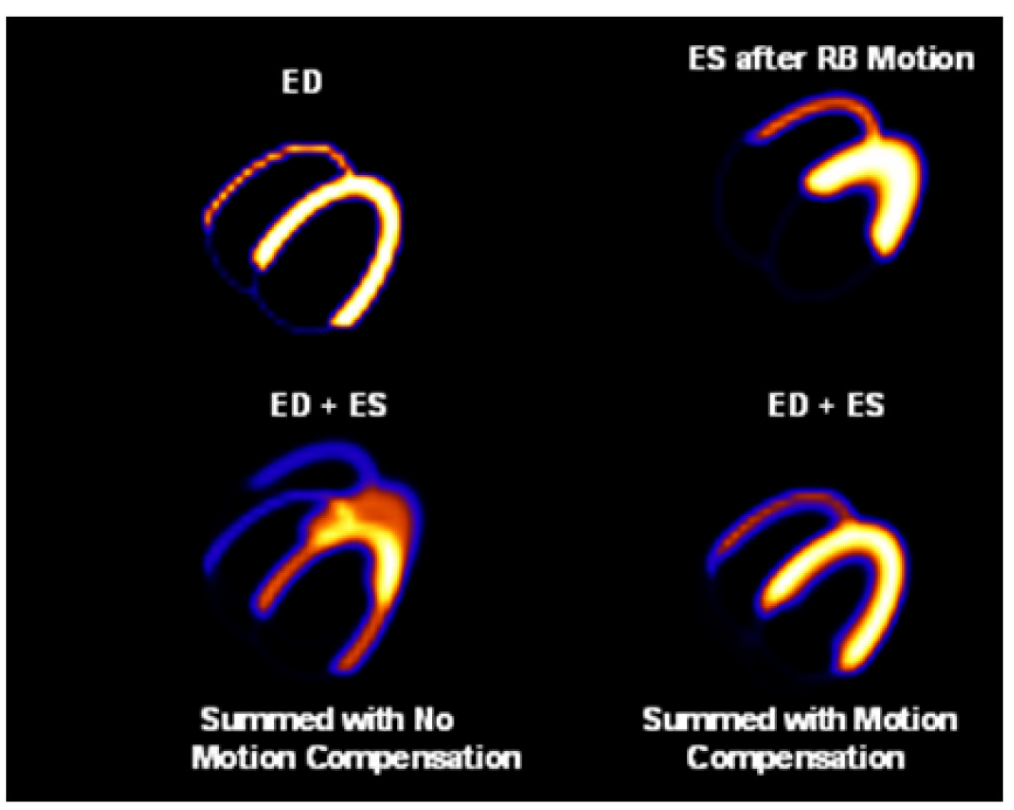

Fig. 6.

In simulations with the gated MCAT phantom, the end-diastolic (ED) (Upper left) and the endsystolic (ES) (Upper right) images were used. The ES image was translated and rotated (Upper right), and summed into the ED image without compensation for the motion (Lower left), and with compensation for the motion estimated from the generalized center-of-mass points (Lower right). Note the full 3D heart was employed in this study. Translation and rotation were also 3D. Thus the summed ED + ES slice at lower right is not the sum of the ED and ES slices shown at the top of the figure, but the sum of the ED slice at top and its appropriate counter part after 3D translation and rotation. 


\section{Table 1}

In the MCAT phantom simulations, the motion was estimated from images for 4 noise-levels, using the smoothing function and three generalized center-of-mass points determined to minimize the RMSD (root-mean-square difference). Note ROT_Y is Y-axis rotation.

\begin{tabular}{|c|c|c|c|c|}
\hline $\begin{array}{l}\text { Optimal Parameters } \\
\text { \& Estimated Motion }\end{array}$ & $\begin{array}{c}\text { Noise- } \\
\text { Free }\end{array}$ & \begin{tabular}{|l|}
$1 \mathrm{M}$ \\
Counts
\end{tabular} & $\begin{array}{l}0.5 \mathrm{M} \\
\text { Counts }\end{array}$ & $\begin{array}{l}0.1 \mathrm{M} \\
\text { Counts }\end{array}$ \\
\hline $\begin{array}{c}\begin{array}{c}\text { Optimal Smoothing } \\
\text { Parameter }\end{array} \\
\end{array}$ & $\sigma=2.0$ & $\sigma=2.5$ & $\sigma=1.5$ & $\sigma=5.0$ \\
\hline $\begin{array}{c}\text { Optimal Power } \\
\text { Function Indexes }\end{array}$ & $3,4,5$ & $1,2,3$ & $1,2,3$ & $1,2,3$ \\
\hline $\begin{array}{c}\text { Minimum Relative } \\
\text { RMSD } \\
\left(10^{-6}\right) \\
\end{array}$ & 0.0578 & 0.213 & 0.279 & 0.567 \\
\hline $\begin{array}{c}\text { Translations }-\Delta \mathrm{x}, \\
\Delta \mathrm{y}, \Delta \mathrm{z}(\mathrm{cm})\end{array}$ & $\begin{array}{c}1.96,3.01, \\
2.50 \\
\end{array}$ & $\begin{array}{c}2.00,3.16, \\
2.60 \\
\end{array}$ & $\begin{array}{c}1.99,3.10 \\
2.54 \\
\end{array}$ & $\begin{array}{c}1.58,3.27 \\
2.60 \\
\end{array}$ \\
\hline ROT Y (deg) & 14.6 & 14.4 & 15.8 & 12.0 \\
\hline
\end{tabular}


Table 2

In the MCAT phantom simulations, the motion was estimated from images for 4 noise-levels, using the principle-axes. Note RMSD is root-mean-square difference and ROT_Y is Y-axis rotation.

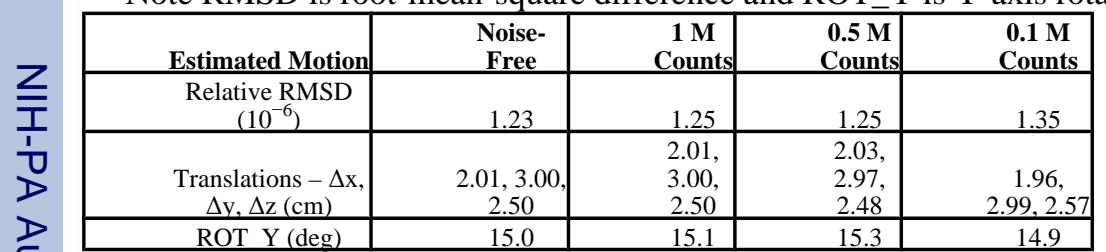

True Motion: (Horizontal) $\Delta \mathrm{x}=2 \mathrm{~cm}$, (Vertical) $\Delta \mathrm{y}=3 \mathrm{~cm}$, (Axial) $\Delta \mathrm{z}=2.5 \mathrm{~cm}$. Rotation by (Vertical) Y-axis $=15 \mathrm{deg}$. 
Table 3

The relative mean-square error (RMSE) as calculated for the images in the middle and lower rows in Fig. 2.

\begin{tabular}{|c|r|r|r|r|}
\hline \multirow{2}{*}{ Methods } & \multicolumn{4}{|c|}{ Relative RMSE $=$ RMSE/Counts } \\
\cline { 2 - 5 } & Noise-free & $\mathbf{1 ~ M}$ & $\mathbf{0 . 5} \mathbf{~}$ & $\mathbf{0 . 1} \mathbf{M}$ \\
\hline $\begin{array}{c}\text { No Motion } \\
\text { Compensation }\end{array}$ & 0.592 & 0.603 & 0.602 & 0.677 \\
\hline $\begin{array}{c}\text { Motion } \\
\text { Compensation }\end{array}$ & 0.034 & 0.105 & 0.114 & 0.282 \\
\hline
\end{tabular}




\section{Table 4}

The rigid-body motions of the anthropomorphic phantom were estimated from images reconstructed from the four datasets, by using the generalized center-of-mass points and the principle-axes, and compared with the motions detected by Polaris.

\begin{tabular}{|c|c|c|c|c|}
\hline$Z$ & $\begin{array}{c}\text { Dataset } \\
\#\end{array}$ & $\begin{array}{c}\text { Motion by } \\
\text { Polaris }\end{array}$ & $\begin{array}{c}\text { Motion by the } \\
\text { Generalized } \\
\text { Center-of-Mass } \\
\text { Points } \\
\end{array}$ & $\begin{array}{l}\text { Motion by the } \\
\text { Principle-Axes } \\
\text { Method }\end{array}$ \\
\hline $\begin{array}{l}I \\
\text { I } \\
I\end{array}$ & 2 & $\begin{array}{c}-6.19 \mathrm{~cm} \\
\text { translation by } \\
\text { axial }(Z) \\
\text { direction } \\
\end{array}$ & $\begin{array}{c}-6.18 \mathrm{~cm} \\
\text { translation by } \\
\text { axial }(\mathrm{Z}) \text { direction }\end{array}$ & $\begin{array}{c}-6.09 \mathrm{~cm} \\
\text { translation by } \\
\text { axial }(\mathrm{Z}) \text { direction }\end{array}$ \\
\hline$=$ & 3 & $\begin{array}{c}6.91 \text { degree } \\
\text { rotation by } \\
\text { vertical (Y) axis }\end{array}$ & $\begin{array}{c}8.04 \text { degree } \\
\text { rotation by vertical } \\
(\mathrm{Y}) \text { axis }\end{array}$ & $\begin{array}{c}7.74 \text { degree } \\
\text { rotation by } \\
\text { vertical (Y) axis }\end{array}$ \\
\hline @ & 4 & $\begin{array}{c}-15.6 \text { degree } \\
\text { rotation by } \\
\text { horizontal }(\mathrm{X}) \\
\text { axis } \\
\end{array}$ & $\begin{array}{c}-16.4 \text { degree } \\
\text { rotation by } \\
\text { horizontal }(\mathrm{X}) \text { axis }\end{array}$ & $\begin{array}{c}-17.2 \text { degree } \\
\text { rotation by } \\
\text { horizontal }(\mathrm{X}) \\
\text { axis } \\
\end{array}$ \\
\hline
\end{tabular}

